\title{
Remarks on the Dynamical Mass Generation in Confining Yang-Mills Theories
}

\author{
S. P. Sorella \\ Departamento de Física Teórica, Instituto de Física, Universidade do Estado do Rio de Janeiro, \\ Rua São Francisco Xavier 524, 20550-013, Maracanã, Rio de Janeiro, Brazil
}

Received on 9 December, 2005

\begin{abstract}
The dynamical mass generation for gluons is discussed in Euclidean Yang-Mills theories supplemented with a renormalizable mass term. The mass parameter is not free, being determined in a self-consistent way through a gap equation which obeys the renormalization group. The example of the Landau gauge is worked out explicitly at one loop order. A few remarks on the issue of the unitarity are provided.
\end{abstract}

Keywords: Yang-Mills theories; Confinement

\section{INTRODUCTION}

In the last years many efforts have been done to put forward the idea that gluons might acquire a mass through a dynamical mechanism. These efforts have led to a considerable amount of evidence, obtained through theoretical and phenomenological studies [1-22] as well as from lattice simulations [23-33]. Many aspects related to the dynamical gluon mass generation deserve a better understanding. This is the case, for example, of the unitarity of the resulting theory, a highly nontrivial topic, due to the confining character of $Q C D$.

Needless to say, the unitarity of the $S$ matrix is a fundamental property of the spectrum of a quantum field theory. It expresses the conservation of the probability of the amplitudes corresponding to the various scattering processes among the excitations of the spectrum.

In a nonconfining theory, the first step in the construction of the $S$ matrix is the introduction of the so-called $|i n\rangle$ and $\mid$ out $\rangle$ Fock spaces characterizing the asymptotic behavior of the physical states before, $t \rightarrow-\infty$, and after, $t \rightarrow+\infty$, a scattering process. The $S$-matrix is thus defined as the unitary operator which interpolates between the spaces $\mid$ in $\rangle$ and $\mid$ out $\rangle$, namely

$$
\mid \text { in }\rangle=S \mid \text { out }\rangle \text {. }
$$

The relation of this equation with the Green's functions of the theory is provided by the $L S Z$ formalism. A key ingredient of this formalism is the introduction of the asymptotic fields, $\varphi_{\text {in }}$ and $\varphi_{\text {out }}$, describing the asymptotic behavior of the interacting fields $\varphi$, according to

$$
\begin{aligned}
\left.\varphi\right|_{t \rightarrow-\infty} & =Z^{1 / 2} \varphi_{\text {in }}, \\
\left.\varphi\right|_{t \rightarrow+\infty} & =Z^{1 / 2} \varphi_{\text {out }} .
\end{aligned}
$$

The asymptotic fields $\varphi_{\text {in }}$ and $\varphi_{\text {out }}$ allow us to define the creation and annihilation operators $\left(a_{\text {in }}^{\dagger}, a_{\text {in }}\right)$ and $\left(a_{\text {out }}^{\dagger}, a_{\text {out }}\right)$, from which the Fock spaces $\mid$ in $\rangle$ and $\mid$ out $\rangle$ are obtained. The entire construction relies on the possibility that the asymptotic fields can be consistently introduced.

In a confining theory like $Q C D$, the quanta associated with the basic fields of the theory, i.e. the gluon field $A_{\mu}^{a}$ and the quark fields $\psi, \bar{\psi}$, cannot be observed as free particles, due to color confinement. The physical spectrum of the theory is made up by colorless bound states of quarks and gluons giving rise, for instance, to barions, mesons and glueballs. This implies that the asymptotic Fock spaces $\mid$ in $\rangle, \mid$ out $\rangle$ of the theory have to be defined through suitable operators from which the physical spectrum of the excitations is constructed. Of course, the $S$-matrix describing the scattering amplitudes among the excitations of the physical spectrum of the theory has to be unitary. Although intuitively simple and easily understandable, this framework is far beyond our present capabilities. An operational definition of the gauge invariant colorless operators defining the physical spectrum of the excitations and a well defined set of rules to evaluate their scattering amplitudes are not yet at our disposal.

Quantized Yang-Mills theories are described by the Faddeev-Popov Lagrangian [38]

$$
\begin{gathered}
S=S_{Y M}+S_{g f}= \\
\mathrm{Z} d^{4} x\left(\frac{1}{4} F_{\mu \nu}^{a} F_{\mu \nu}^{a}+b^{a} \partial_{\mu} A_{\mu}^{a}+\bar{c}^{a} \partial_{\mu}\left(D_{\mu} c\right)^{a}\right),
\end{gathered}
$$

here taken in the Landau gauge. The field $b^{a}$ in expression (3) is the Lagrange multiplier enforcing the Landau gauge condition, $\partial_{\mu} A_{\mu}^{a}=0$, while $\bar{c}^{a}, c^{a}$ stand for the Faddeev-Popov ghosts. The action (3) is renormalizable to all orders of perturbation theory and displays color confinement[39]. Furthermore, thanks to the asymptotic freedom, the gauge field $A_{\mu}^{a}$ behaves almost freely at very high energies, where perturbation theory is reliable. However, at low energies, the coupling constant increases and the effects of color confinement cannot be neglected. We do have thus a good understanding of the properties of the field $A_{\mu}^{a}$ at high energies, whereas it becomes more and more difficult to have a clear picture of $A_{\mu}^{a}$, and of the whole theory, as the energy decreases. We might thus adopt the point of view of starting with a renormalizable action built up with a gauge field $A_{\mu}^{a}$ which accommodates the largest possible number of degrees of freedom. This would amount to start with a quantized massive Yang-Mills action

$$
S_{m}=S_{Y M}+S_{g f}+S_{\text {mass }},
$$

where $S_{\text {mass }}$ is a suitable mass term for the gauge field $A_{\mu}^{a}$. The best choice for $S_{\text {mass }}$ would be a gauge invariant, renormalizable local mass term. However, no local renormalizable 
gauge invariant mass term built up with gauge fields only is at our disposal. Nevertheless, it might be worth reminding that, recently, a consistent framework for the nonlocal gauge invariant mass operator

$$
O(A)=-\frac{1}{2}{ }^{\mathrm{Z}} d^{4} x F_{\mu \nu}^{a}\left[\left(D^{2}\right)^{-1}\right]^{a b} F_{\mu \nu}^{b} .
$$

has been achieved [34]. More precisely, the nonlocal operator (5) can be cast in local form by means of the introduction of a suitable set of additional fields. The resulting local the- ory displays the important property of being multiplicatively renormalizable [34].

Though, for the time being, we give up of the requirement of the gauge invariance. This will enable us to present our analysis with the help of a relatively simple example. Therefore, as possible mass term we shall take

$$
S_{\text {mass }}=\frac{1}{2} m^{2} \quad d^{4} x A_{\mu}^{a} A_{\mu}^{a},
$$

so that

$$
S_{m}=d^{4} x\left(\frac{1}{4} F_{\mu \nu}^{a} F_{\mu \nu}^{a}+\frac{1}{2} m^{2} A_{\mu}^{a} A_{\mu}^{a}+b^{a} \partial_{\mu} A_{\mu}^{a}+\bar{c}^{a} \partial_{\mu}\left(D_{\mu} c\right)^{a}\right)
$$

Expression (7) provides an example of a massive nonabelian gauge theory which is renormalizable to all orders of perturbation theory [35], while obeying the renormalization group equations (RGE).

A few remarks are now in order:

- The amplitudes corresponding to the scattering processes among gluons and quarks display now a violation of the unitarity. This can be understood by noting that the inclusion of the mass term $m^{2} A_{\mu}^{a} A_{\mu}^{a}$ gives rise to a $B R S T$ operator which is not nilpotent. However, as shown in [35], it is still possible to write down suitable Slavnov-Taylor identities which ensure that the massive theory (7) is renormalizable to all orders of perturbation theory. Moreover, if sufficiently small, this violation of the unitarity might not be in conflict with the confining character of the theory. Otherwise said, since gluons are not directly observable, we could allow for a gauge field $A_{\mu}^{a}$ with the largest possible number of degrees of freedom, provided the renormalizability is preserved and one is able to recover the results of the massless case at very high energies.

- This framework would be useless if the value of the mass parameter $m$ would be free, meaning that we are introducing a new arbitrary parameter in the theory, thereby changing its physical meaning. A different situation is attained by demanding that the mass parameter is determined in a self-consistent way as a function of the coupling constant $g$. This can be obtained by requiring that the mass $m$ in eq.(7) is a solution of a suitable gap equation. In other words, even if the mass $m$ is included in the starting gauge-fixed theory, it does not play the role of a free parameter, as it is determined once the quantum effects are properly taken into account. Here, we rely on the lack of an exact description of a confining Yang-Mills theory at low energies. We start then with the largest possible number of degrees of freedom compatible with the renormalizability requirement and fix the mass parameter through the gap equation. If the resulting value of $m$ will be small enough, one can argue that the unitarity is violated by terms which become less and less important as the energy of the process increases, so that the amplitudes of the massless case are in practice recovered at very high energies. The present set up might thus provide a different characterization of the aforementioned phenomenon of the dynamical gluon mass generation, which has already been successfully described in $[1,7,10,11,13,14,16,17]$. In the next section, the gap equation for the mass $m$ will be discussed.

\section{THE GAP EQUATION FOR THE MASS PARAMETER $m$}

The gap equation for the mass parameter $m$ is obtained by requiring that the vacuum functional $\mathcal{E}$ defined by

$$
e^{-V \mathcal{E}}=[D \Phi] e^{-\left(S_{m}+V \eta(g) \frac{m^{4}}{2}\right)}
$$

where $V$ is the Euclidean space-time volume, obeys a minimization condition with respect to the mass $m$, i.e. the value of the mass $m$ is determined by demanding that it corresponds to the minimum of the vacuum functional $\mathcal{E}$, namely

$$
\frac{\partial \mathcal{E}}{\partial m^{2}}=0
$$

Equation (9) is the gap equation for the mass parameter $m$. The quantity $\eta(g)$ in eq.(8) is a dimensionless parameter whose loop expansion

$$
\eta(g)=\eta_{0}(g)+\hbar \eta_{1}(g)+\hbar^{2} \eta_{2}(g)+\ldots .
$$

accounts for the quantum effects related to the renormalization of the vacuum diagrams in the massive case. The parameter $\eta(g)$ can be obtained order by order by requiring that the vacuum functional $\mathcal{E}$ obeys the renormalization group equations 
(RGE)

$$
\mu \frac{d \mathcal{E}}{d \mu}=0
$$

meaning that $\mathcal{E}$ is independent from the renormalization scale $\mu$, as it will be explicitly verified in the next section. Equation (11) expresses an important property of the vacuum functional $\mathcal{E}$. We also remark that a term of the kind of $\eta m^{4}$ in eq.(8) has been already obtained[40] in [1] in the evaluation of the vacuum energy of Yang-Mills theories when gluons are massive.

The gap equation equation (11) can be given a simple interpretation. Due to the lack of an exact description of Yang-Mills theories at low energies, we have adopted the point of view of starting with a renormalizable massive action, as given in eq.(7). As far as the mass parameter $m$ is free, expression (7) can be interpreted as describing a family of massive models, parametrized by $m$. For each value of $m$ we have a specific renormalizable model. Moreover, as the introduction of a mass term has an energetic coast, we might figure out that, somehow, the dynamics will select precisely that model corresponding to the lowest energetic coast, as expressed by the gap equation (11).

Before starting with explicit calculations let us summarize our point of view:

- Since gluons are not directly observable, we allow for a gauge field $A_{\mu}^{a}$ with the largest number of degrees of freedom compatible with the requirement of renormalizability.

- This amounts to start with a renormalizable massive action, as given in eq. (7). However, the mass parameter $m$ is determined in a self-consistent way by imposing the minimizing condition $(9)$ on the vacuum functional $\mathcal{E}$.

- Also, it is worth observing that, in the case of the massive model of eq. (7), a non vanishing solution, $m_{\text {sol }}^{2} \neq 0$, of the gap equation (9) implies the existence of a non vanishing dimension two gluon condensate $\left\langle A_{\mu}^{a} A_{\mu}^{a}\right\rangle$. In fact, differentiating equation (8) with respect to $m^{2}$ and setting $m^{2}=m_{\text {sol }}^{2}$, one obtains

$$
\frac{1}{2}\left\langle A_{\mu}^{a} A_{\mu}^{a}\right\rangle=-\eta m_{\text {sol }}^{2} .
$$

\section{EVALUATION OF THE VACUUM FUNCTIONAL $\mathcal{E}$ AT ONE LOOP ORDER}

In the case of pure $S U(N)$ Yang-Mills theories, for the vacuum functional $\mathcal{E}$ we have

$$
e^{-V \mathcal{E}}=[D \Phi] e^{-\left(S_{m}+V \eta \frac{m^{4}}{2}\right)},
$$

with $S_{m}$ given by expression (7), namely

$$
S_{m}={ }^{\mathrm{Z}} d^{4} x\left(\frac{1}{4} F_{\mu \nu}^{a} F_{\mu \nu}^{a}+\frac{1}{2} m^{2} A_{\mu}^{a} A_{\mu}^{a}+b^{a} \partial_{\mu} A_{\mu}^{a}+\bar{c}^{a} \partial_{\mu}\left(D_{\mu} c\right)^{a}\right) .
$$

As it has been proven in [35], the massive action (14) is multiplicatively renormalizable to all orders of perturbation theory. In particular, for the mass renormalization we have [35]

$$
\begin{aligned}
g_{0} & =Z_{g} g, \\
A_{0} & =Z_{A}^{1 / 2} A \\
m_{0}^{2} & =Z_{m^{2}} m^{2}, \\
Z_{m^{2}} & =Z_{g} Z_{A}^{-1 / 2},
\end{aligned}
$$

from which the running of the mass $m^{2}$ is easily deduced

$$
\mu \frac{\partial m^{2}}{\partial \mu}=-\gamma_{m^{2}} m^{2}
$$

with

$$
\begin{gathered}
\gamma_{m^{2}}\left(g^{2}\right)=\gamma_{0} g^{2}+\bar{\gamma}_{1} g^{4}+O\left(g^{6}\right) \\
\gamma_{0}=\frac{35}{6} \frac{N}{16 \pi^{2}}, \quad \bar{\gamma}_{1}=\frac{449}{24}\left(\frac{N}{16 \pi^{2}}\right)^{2} .
\end{gathered}
$$

Also

$$
\begin{gathered}
\beta\left(g^{2}\right)=\bar{\mu} \frac{\partial g^{2}}{\partial \bar{\mu}}=-2\left(\beta_{0} g^{4}+\beta_{1} g^{6}+O\left(g^{8}\right)\right), \\
\beta_{0}=\frac{11}{3} \frac{N}{16 \pi^{2}}, \quad \beta_{1}=\frac{34}{3}\left(\frac{N}{16 \pi^{2}}\right)^{2} .
\end{gathered}
$$

In order to obtain the parameter $\eta$ at one-loop order, it is useful to note that expression (13) can be written in localized form as

$$
\begin{aligned}
& e^{-V \mathcal{E}}={ }^{\mathrm{Z}}[D \Phi] e^{-\left(S_{m}+V \eta \frac{m^{4}}{2}\right)}, \\
& { }^{\mathrm{Z}} D J(x) \delta\left(J(x)-m^{2}\right) e^{-W(J)},
\end{aligned}
$$


with

$$
\begin{aligned}
e^{-W(J)} & =\quad[D \Phi] e^{-S(J)}, \\
S(J) & =S_{Y M}+S_{g f}+d^{4} x\left(\frac{1}{2} J(x) A_{\mu}^{a} A_{\mu}^{a}+\frac{\eta}{2} J^{2}(x)\right) .
\end{aligned}
$$

From equation (21) it follows that the renormalization of the vacuum functional $\mathcal{E}$ can be achieved by renormalizing the functional $W(J)$ in the presence of the local source $J(x)$, and then set $J=m^{2}$ at the end. The renormalization of the functional $W(J)$ has been worked out at two-loops in [7]. By simple inspection, it turns out that the parameter $\eta$ is related to the LCO parameter $\zeta$ of [7] by $\eta=-\zeta$, yielding

$$
\eta=-\frac{9}{13 g^{2}} \frac{N^{2}-1}{N}-\hbar \frac{161}{52} \frac{N^{2}-1}{16 \pi^{2}}+O\left(g^{2}\right) .
$$

Thus, for the vacuum functional $\mathcal{E}$ at one-loop order in the $\overline{M S}$ scheme, we get

$$
\begin{gathered}
\mathcal{E}=\frac{m^{4}}{2}\left(-\frac{9}{13 g^{2}} \frac{N^{2}-1}{N}-\hbar \frac{161}{52} \frac{N^{2}-1}{16 \pi^{2}}\right)+ \\
3 \hbar \frac{N^{2}-1}{64 \pi^{2}} m^{4}\left(-\frac{5}{6}+\log \frac{m^{2}}{\bar{\mu}^{2}}\right)
\end{gathered}
$$

where we have introduced the factor $\hbar$ to make clear the order of the various terms. It is useful to check explicitly that the above expression obeys the RGE equations. Indeed, from eqs.(17), (19) we obtain

$$
\begin{aligned}
\bar{\mu} \frac{d \mathcal{E}}{d \bar{\mu}} & =-\hbar \gamma_{0} g^{2} m^{4}\left(-\frac{9}{13 g^{2}} \frac{N^{2}-1}{N}\right)+\hbar \frac{m^{4}}{2} \frac{9}{13 g^{4}} \frac{N^{2}-1}{N}\left(-2 \beta_{0} g^{4}\right)-\hbar 6 \frac{N^{2}-1}{64 \pi^{2}} m^{4}+O\left(\hbar^{2}\right) \\
& =\hbar m^{4} \frac{N^{2}-1}{16 \pi^{2}}\left(\frac{35}{6}\right) \frac{9}{13}-\hbar m^{4} \frac{N^{2}-1}{16 \pi^{2}} \frac{33}{13}-\hbar 6 \frac{N^{2}-1}{64 \pi^{2}} m^{4}+O\left(\hbar^{2}\right) \\
& =\hbar m^{4} \frac{N^{2}-1}{16 \pi^{2}}\left(\frac{35}{6} \frac{9}{13}-\frac{33}{13}-\frac{6}{4}\right)+O\left(\hbar^{2}\right)=\hbar m^{4} \frac{N^{2}-1}{16 \pi^{2}}\left(\frac{105}{26}-\frac{33}{13}-\frac{3}{2}\right)+O\left(\hbar^{2}\right) \\
& =\hbar m^{4} \frac{N^{2}-1}{16 \pi^{2}}\left(\frac{105-66-39}{26}\right)+O\left(\hbar^{2}\right)=O\left(\hbar^{2}\right) .
\end{aligned}
$$

It remains now to look for a sensible solution of the gap equation (9). This will be the task of the next section.

\section{A. Searching for a sensible minimum}

In order to search for a sensible solution of the gap equation (9), $\frac{\partial \mathcal{E}}{\partial m^{2}}=0$, we first remove the freedom existing in the renormalization of the mass parameter by replacing it with a renormalization scheme and scale independent quantity. This can be achieved along the lines outlined in [10] in the analysis of the gluon condensate $\left\langle A_{\mu}^{a} A_{\mu}^{a}\right\rangle$ within the 2PPI expansion technique. Let us first change notation

$$
\begin{gathered}
g^{2} \rightarrow \bar{g}^{2}, \\
m^{2} \rightarrow \bar{m}^{2},
\end{gathered}
$$

and rewrite the one-loop vacuum functional as

$$
\mathcal{E}=\frac{9}{13} \frac{N^{2}-1}{N} \frac{1}{\bar{g}^{2}}\left[-\frac{\bar{m}^{4}}{2}+\frac{13}{3} \frac{N \bar{g}^{2}}{64 \pi^{2}} \bar{m}^{4}\left(\log \frac{\bar{m}^{2}}{\bar{\mu}^{2}}-\frac{113}{39}\right)\right] .
$$

As done in [10], we introduce the scheme and scale independent quantity $\widetilde{m}^{2}$ through the relation

$$
\widetilde{m}^{2}=\bar{f}\left(\bar{g}^{2}\right) \bar{m}^{2} \text {. }
$$

From

$$
\bar{\mu} \frac{\partial \bar{m}^{2}}{\partial \bar{\mu}}=-\bar{\gamma}_{m^{2}}\left(\bar{g}^{2}\right) \bar{m}^{2}
$$

with

$$
\bar{\gamma}_{m^{2}}\left(\bar{g}^{2}\right)=\gamma_{0} \bar{g}^{2}+\bar{\gamma}_{1} \bar{g}^{4}+O\left(\bar{g}^{6}\right)
$$

$$
\gamma_{0}=\frac{35}{6} \frac{N}{16 \pi^{2}}, \quad \bar{\gamma}_{1}=\frac{449}{24}\left(\frac{N}{16 \pi^{2}}\right)^{2}
$$


we obtain the condition

$$
\bar{\mu} \frac{\partial \bar{f}\left(\bar{g}^{2}\right)}{\partial \bar{\mu}}=\bar{\gamma}_{m^{2}}\left(\bar{g}^{2}\right) \bar{f}\left(\bar{g}^{2}\right)
$$

from which it follows that

$$
\bar{\mu} \frac{\partial \widetilde{m}^{2}}{\partial \bar{\mu}}=0 .
$$

Equation (32) is easily solved, yielding

$$
\begin{aligned}
\bar{f}\left(\bar{g}^{2}\right) & =\left(\bar{g}^{2}\right)^{-\frac{\gamma_{0}}{2 \beta_{0}}}\left(1+f_{0} \bar{g}^{2}+O\left(\bar{g}^{4}\right)\right), \\
f_{0} & =\frac{1}{2 \beta_{0}}\left(\frac{\gamma_{0}}{\beta_{0}} \beta_{1}-\bar{\gamma}_{1}\right),
\end{aligned}
$$

where the coefficients $\beta_{0}, \beta_{1}$ are given in eqs.(19), (20). Moreover, one has to take into account that a change of scheme entails a change in the coupling constant $\bar{g}^{2}$, according to

$$
\bar{g}^{2}=g^{2}\left(1+b_{0} g^{2}+O\left(g^{4}\right)\right) .
$$

The coefficient $b_{0}$ in eq.(35) expresses the freedom related to the choice of the renormalization scheme. It will be fixed by demanding that the coupling constant is renormalized in such a scheme so that the vacuum functional $\mathcal{E}$ takes the form

$$
\mathcal{E}\left(\widetilde{m}^{2}\right)=\frac{9}{13} \frac{N^{2}-1}{N} \frac{1}{\left(g^{2}\right)^{1-\frac{\gamma_{0}}{\beta_{0}}}}\left(-\frac{\widetilde{m}^{4}}{2}+\widetilde{m}^{4} \frac{N g^{2}}{16 \pi^{2}} E_{1} L\right)
$$

where $L$ stands for

$$
L=\log \frac{\widetilde{m}^{2}\left(g^{2}\right)^{\frac{\gamma_{0}}{2 \beta_{0}}}}{\bar{\mu}^{2}}
$$

and $E_{1}$ is a numerical coefficient. After a simple calculation, we get

$$
\mathcal{E}=\frac{9}{13} \frac{N^{2}-1}{N} \frac{1}{\left(g^{2}\right)^{1-\frac{\gamma_{0}}{\beta_{0}}}}\left[-\frac{\widetilde{m}^{4}}{2}+\widetilde{m}^{4} \frac{13}{3} \frac{N g^{2}}{64 \pi^{2}}\left(L-\frac{113}{39}+\frac{3}{13} \frac{64 \pi^{2}}{N}\left(f_{0}+\frac{b_{0}}{2}\left(1-\frac{\gamma_{0}}{\beta_{0}}\right)\right)\right)\right]
$$

Therefore, for $b_{0}$ one has

$$
-\frac{113}{39}+\frac{3}{13} \frac{64 \pi^{2}}{N}\left(f_{0}+\frac{b_{0}}{2}\left(1-\frac{\gamma_{0}}{\beta_{0}}\right)\right)=0
$$

namely

$$
b_{0}=-\frac{4331}{396} \frac{N}{16 \pi^{2}} .
$$

For the vacuum functional $\mathcal{E}\left(\widetilde{m}^{2}\right)$ one gets

$$
\mathcal{E}=\frac{9}{13} \frac{N^{2}-1}{N} \frac{1}{\left(g^{2}\right)^{1-\frac{\gamma_{0}}{\beta_{0}}}}\left[-\frac{\widetilde{m}^{4}}{2}+\widetilde{m}^{4} \frac{13}{3} \frac{N g^{2}}{64 \pi^{2}} L\right] .
$$

In terms of the scale independent variable $\widetilde{m}^{2}$, the gap equation reads

$$
\frac{\partial \mathcal{E}}{\partial \widetilde{m}^{2}}=0
$$

so that

$$
-\widetilde{m}^{2}+\widetilde{m}^{2} \frac{26}{3} \frac{N g^{2}}{64 \pi^{2}} L+\widetilde{m}^{2} \frac{13}{3} \frac{N g^{2}}{64 \pi^{2}}=0 .
$$

Next to the solution, $\widetilde{m}^{2}=0$, we have the nontrivial solution $\widetilde{m}_{\text {sol }}$ given by

$$
-1+\frac{26}{3} \frac{N g^{2}}{64 \pi^{2}} \log \left(\frac{\widetilde{m}_{\text {sol }}^{2}\left(g^{2}\right)^{\frac{\gamma_{0}}{2 \beta_{0}}}}{\bar{\mu}^{2}}\right)+\frac{13}{3} \frac{N g^{2}}{64 \pi^{2}}=0 .
$$

In order to find a sensible solution of this equation, a suitable choice of the scale $\bar{\mu}$ has to be done. Here, we take full advantage of the RGE invariance of the vacuum functional $\mathcal{E}$, and set

$$
\bar{\mu}^{2}=\tilde{m}_{s o l}^{2}\left(g^{2}\right)^{\frac{\gamma_{0}}{2 \beta_{0}}} e^{-s},
$$

where $s$ is an arbitrary parameter which will be chosen at our best convenience. The possibility of introducing this parameter relies on the independence of the vacuum functional $\mathcal{E}$ from the renormalization scale $\bar{\mu}$. Furthermore, recalling that

$$
g^{2}(\bar{\mu})=\frac{1}{\beta_{0} \log \frac{\bar{\mu}^{2}}{\Lambda^{2}}},
$$

and that, due to the change of the renormalization scheme,

$$
\Lambda^{2}=\Lambda_{\overline{M S}}^{2} e^{-\frac{b_{0}}{\beta_{0}}}
$$

for the effective coupling and the mass $\widetilde{m}_{s o l}$, one finds

$$
\left.\frac{N g^{2}}{16 \pi^{2}}\right|_{1-\text { loop }}=\frac{12}{13} \frac{1}{(1+2 s)},
$$

$\left.\widetilde{m}_{\text {sol }}\right|_{1-\text { loop }}=\left(\frac{12}{13} \frac{16 \pi^{2}}{N} \frac{1}{(1+2 s)}\right)^{-\frac{\gamma_{0}}{4 \beta_{0}}} e^{-\frac{b_{0}}{2 \beta_{0}}} e^{\frac{13}{88}(1+2 s)} e^{\frac{s}{2}} \Lambda_{\overline{M S}}$ 
Therefore, choosing $s=0.6$, and setting $N=3$, the following one-loop estimates are found

$$
\begin{aligned}
\left.\frac{N g^{2}}{16 \pi^{2}}\right|_{1-\text { loop }} \simeq 0.42 \\
\left.\widetilde{m}_{\text {sol }}\right|_{1-\text { loop }} \simeq 2.4 \Lambda_{\overline{M S}} \simeq 560 \mathrm{MeV}, \\
\Lambda_{\overline{M S}} \simeq 233 \mathrm{MeV} \\
\left.\sqrt{\left\langle A_{\mu}^{a} A_{\mu}^{a}\right\rangle}\right|_{1-\text { loop }} ^{N=3} \simeq 0.22 \mathrm{GeV},
\end{aligned}
$$

and

$$
\left.\mathcal{E}\left(\widetilde{m}_{\text {sol }}\right)\right|_{1-\text { loop }} ^{N=3} \simeq-90 \Lambda_{\overline{M S}}^{4} \simeq-0.265(\mathrm{GeV})^{4} .
$$

Note that the value obtained for $\widetilde{m}_{s o l}$ is close to that already reported for the dynamical gluon mass in the Landau gauge $[7,10,11,17,27,31]$. It should be remarked that the results (50), (51) have been obtained within a one-loop approximation. As such, they can be taken only as a preliminary indication. To find more reliable results, one has to go beyond the one-loop approximation. Nevertheless, these calculations suggest that a non vanishing gluon mass might emerge from the gap equation (9).

\section{CONCLUSION}

In this work the issue of the dynamical mass generation for gluons has been addressed. Due to color confinement, gluons are not observed as free particles. Thanks to the asymptotic freedom, the gauge field $A_{\mu}^{a}$ behaves almost freely at very high energies, where we have a good understanding of its properties. However, as the energy decreases the effects of confinement cannot be neglected and it becomes more and more difficult to have a clear understanding of $A_{\mu}^{a}$. As a consequence, one does not exactly know what is the correct starting point in the low energy region. We might thus adopt the point of view of starting with a renormalizable action built up with a gauge field $A_{\mu}^{a}$ which accommodates the largest possible number of degrees of freedom. This would amount to take as starting point a renormalizable massive action as considered, for example, in expression (7). The mass parameter $m$ is not treated as a free parameter. Instead it is determined by a gap equation, eq.(9), obtained by minimizing the vacuum functional $\mathcal{E}$ of eq.(8) with respect to the mass parameter $m$. A preliminary analysis of this gap equation at one-loop shows that a nonvanishing gluon mass might emerge. Also, the vacuum functional $\mathcal{E}$ displays the important feature of obeying the renormalization group equations.

Finally, we underline that the infrared behavior of the gluon propagator is expected to be affected by several mass parameters, with different origins. For instance, as pointed out in $[36,37]$ in the case of the Landau gauge, the gluon propagator turns out to be affected by both dynamical gluon mass $m$ and Gribov parameter $\gamma$, which arises from the restriction of the domain of integration in the Feynman path integral up to the first Gribov horizon. More precisely, these parameters give rise to a three level gluon propagator which exhibits infrared suppression [36, 37], namely

$$
\begin{gathered}
\left\langle A_{\mu}^{a}(k) A_{v}^{b}(-k)\right\rangle= \\
\delta^{a b}\left(\delta_{\mu v}-\frac{k_{\mu} k_{v}}{k^{2}}\right) \frac{k^{2}}{k^{4}+m^{2} k^{2}+\gamma^{4}} .
\end{gathered}
$$

\section{Acknowledgments}

I am indebted to my friends and colleagues D. Dudal, J. A. Gracey, and H. Verschelde for many valuable discussions. T. Turner and L. R. de Freitas are gratefully acknowledged. We thank the Conselho Nacional de Desenvolvimento Científico e Tecnológico (CNPq-Brazil), the Faperj, Fundação de Amparo à Pesquisa do Estado do Rio de Janeiro, the SR2-UERJ and the Coordenação de Aperfeiçoamento de Pessoal de Nível Superior (CAPES) for financial support.
[1] J. M. Cornwall, Phys. Rev. D 26, 1453 (1982).

[2] J. Greensite and M. B. Halpern, Nucl. Phys. B 271, 379 (1986).

[3] M. Stingl, Phys. Rev. D 34, 3863 (1986) [Erratum-ibid. D 36, 651 (1987)].

[4] M. J. Lavelle and M. Schaden, Phys. Lett. B 208, 297 (1988).

[5] F. V. Gubarev and V. I. Zakharov, Phys. Lett. B 501, 28 (2001) [arXiv:hep-ph/0010096].

[6] F. V. Gubarev, L. Stodolsky and V. I. Zakharov, Phys. Rev. Lett. 86, 2220 (2001) [arXiv:hep-ph/0010057].

[7] H. Verschelde, K. Knecht, K. Van Acoleyen and M. Vanderkelen, Phys. Lett. B 516, 307 (2001) [arXiv:hep-th/0105018].

[8] K. I. Kondo, Phys. Lett. B 514, 335 (2001) [arXiv:hepth/0105299].
[9] K. I. Kondo, T. Murakami, T. Shinohara and T. Imai, Phys. Rev. D 65, 085034 (2002) [arXiv:hep-th/0111256].

[10] D. Dudal, H. Verschelde, R. E. Browne and J. A. Gracey, Phys. Lett. B 562, 87 (2003) [arXiv:hep-th/0302128].

[11] R. E. Browne and J. A. Gracey, JHEP 0311, 029 (2003) [arXiv:hep-th/0306200].

[12] U. Ellwanger and N. Wschebor, Int. J. Mod. Phys. A 18, 1595 (2003) [arXiv:hep-th/0205057].

[13] D. Dudal, H. Verschelde, V. E. R. Lemes, M. S. Sarandy, S. P. Sorella and M. Picariello, Annals Phys. 308, 62 (2003) [arXiv:hep-th/0302168].

[14] D. Dudal, H. Verschelde, J. A. Gracey, V. E. R. Lemes, M. S. Sarandy, R. F. Sobreiro and S. P. Sorella, JHEP 0401, 
044 (2004) [arXiv:hep-th/0311194].

[15] A. C. Aguilar, A. A. Natale and P. S. Rodrigues da Silva, Phys. Rev. Lett. 90, 152001 (2003) [arXiv:hep-ph/0212105].

[16] D. Dudal, J. A. Gracey, V. E. R. Lemes, M. S. Sarandy, R. F. Sobreiro, S. P. Sorella and H. Verschelde, Phys. Rev. D 70, 114038 (2004) [arXiv:hep-th/0406132].

[17] R. E. Browne and J. A. Gracey, Phys. Lett. B 597, 368 (2004) [arXiv:hep-ph/0407238].

[18] J. A. Gracey, Eur. Phys. J. C 39, 61 (2005) [arXiv:hep$\mathrm{ph} / 0411169]$

[19] G. Parisi and R. Petronzio, Phys. Lett. B 94, 51 (1980).

[20] J. H. Field, Phys. Rev. D 66, 013013 (2002) [arXiv:hep$\mathrm{ph} / 0101158]$

[21] A. P. Szczepaniak and E. S. Swanson, Phys. Lett. B 577, 61 (2003) [arXiv:hep-ph/0308268].

[22] X. d. Li and C. M. Shakin, Phys. Rev. D 71, 074007 (2005) [arXiv:hep-ph/0410404].

[23] K. Amemiya and H. Suganuma, Phys. Rev. D 60, 114509 (1999) [arXiv:hep-lat/9811035].

[24] P. Boucaud, A. Le Yaouanc, J. P. Leroy, J. Micheli, O. Pene and J. Rodriguez-Quintero, Phys. Rev. D 63, 114003 (2001) [arXiv:hep-ph/0101302].

[25] P. Boucaud et al., Phys. Rev. D 66, 034504 (2002) [arXiv:hep$\mathrm{ph} / 0203119]$.

[26] P. Boucaud et al., arXiv:hep-lat/0504017.

[27] K. Langfeld, H. Reinhardt and J. Gattnar, Nucl. Phys. B 621, 131 (2002) [arXiv:hep-ph/0107141].

[28] C. Alexandrou, P. de Forcrand and E. Follana, Phys. Rev. D 63,
094504 (2001) [arXiv:hep-lat/0008012].

[29] C. Alexandrou, P. de Forcrand and E. Follana, Phys. Rev. D 65, 114508 (2002) [arXiv:hep-lat/0112043]

[30] V. G. Bornyakov, M. N. Chernodub, F. V. Gubarev, S. M. Morozov and M. I. Polikarpov, Phys. Lett. B 559, 214 (2003) [arXiv:hep-lat/0302002].

[31] E. Ruiz Arriola, P. O. Bowman and W. Broniowski, Phys. Rev. D 70, 097505 (2004) [arXiv:hep-ph/0408309].

[32] T. Suzuki, K. Ishiguro, Y. Mori and T. Sekido, Phys. Rev. Lett. 94, 132001 (2005) [arXiv:hep-lat/0410001].

[33] M. N. Chernodub et al., Phys. Rev. D 72, 074505 (2005) [arXiv:hep-lat/0508004].

[34] M. A. L. Capri, D. Dudal, J. A. Gracey, V. E. R. Lemes, R. F. Sobreiro, S. P. Sorella and H. Verschelde, Phys. Rev. D 72, 105016 (2005) [arXiv:hep-th/0510240].

[35] D. Dudal, H. Verschelde and S. P. Sorella, Phys. Lett. B 555, 126 (2003) [arXiv:hep-th/0212182].

[36] R. F. Sobreiro, S. P. Sorella, D. Dudal and H. Verschelde, Phys. Lett. B 590, 265 (2004) [arXiv:hep-th/0403135].

[37] D. Dudal, R. F. Sobreiro, S. P. Sorella and H. Verschelde, Phys. Rev. D 72, 014016 (2005) [arXiv:hep-th/0502183]

[38] From now on we shall consider pure Yang-Mills theory in the Euclidean space-time.

[39] Although we still lack a definite proof of color confinement, it is doubtless that pure Yang-Mills theory, as given in eq.(3), displays such a phenomenon.

[40] See eq.(6.22) of Sect.VI of [1]. 